\title{
Amelogenin, an extracellular matrix protein, in the treatment of venous leg ulcers and other hard-to-heal wounds: Experimental and clinical evidence
}

\author{
Marco Romanelli' \\ Valentina Dini' \\ Peter Vowden ${ }^{2}$ \\ Magnus S Ågren ${ }^{3}$ \\ 'Department of Dermatology, \\ University of Pisa, Pisa, Italy; \\ ${ }^{2}$ Vascular Unit, Bradford Royal \\ Infirmary, Bradford, United Kingdom; \\ ${ }^{3}$ Department of Surgery K, Bispebjerg \\ Hospitals, Copenhagen University \\ Hospital, Copenhagen, Denmark
}

\begin{abstract}
Amelogenins are extracellular matrix proteins that, under physiological conditions, self-assemble into globular aggregates up to micron-sizes. Studies with periodontal fibroblasts indicate that attachment to these structures increases the endogenous secretion of multiple growth factors and cell proliferation. Pre-clinical and clinical studies indicate that cutaneous wounds benefit from treatment with amelogenins. A randomized controlled trial (RCT) involving patients with hard-to-heal venous leg ulcers (VLUs) (ie, ulcers with a surface area $\geq 10 \mathrm{~cm}^{2}$ and duration of $\geq 6$ months) showed that the application of amelogenin (Xelma ${ }^{\circledR}$, Molnlycke Health Care, Gothenburg, Sweden) as an adjunct treatment to compression results in significant reduction in ulcer size, improvement in the state of ulcers, reduced pain, and a larger proportion of ulcers with low levels of exudate, compared with treatment with compression alone. Amelogenin therapy was also shown to be safe to use in that there were no significant differences in adverse events noted between patients treated with amelogenin plus compression and those treated with compression alone. Case study evaluations indicate that the benefits of amelogenin therapy demonstrated in the RCT are being repeated in "real life" situations and that amelogenin therapy may also have a role to play in the treatment of other wound types such as diabetic foot ulcers.
\end{abstract}

Keywords: extracellular matrix, amelogenin, venous leg ulcers, diabetic foot ulcers, pyoderma gangrenosum

\section{Introduction}

Venous leg ulcers (VLUs) pose a serious clinical dilemma and an economic burden on health services. They are frequently associated with morbidity, pain, and decreased quality of life in affected patients. About $60 \%$ of patients with VLUs will experience pain related to their wounds (Hofman et al 1997), which may be constant or intermittent (Ryan et al 2003). The pain can cause depression and a feeling of constant tiredness (Price et al 2007) and may also interfere with the healing process (Soon and Acton, 2006). These patients may have highly exuding ulcers that could lead to wound malodour and its associated social stigma, resulting in further detrimental psychological effects. VLUs also provide an ideal environment for the growth of pathogenic bacteria, with wound colonisation/infection prevalent in immunocompromised patients. The management of these problems is both time consuming and expensive. VLUs are associated with significant treatment costs (bandages, dressings, and adjunctive therapies) and nursing resource.

About $1 \%-2 \%$ of the whole population (Anderson 2006) and 3\%-5\% of the population over 65 years of age (Mekkes et al 2003) will suffer from a leg ulcer during their lifetime. However, in a typical Western population where the average age is steadily 
increasing, the burden placed upon the health economy by VLUs looks set to increase proportionally. Cost effective treatment of VLUs is therefore vital.

Back in the 1960s, George Winter published his landmark paper in which he demonstrated that, contrary to the belief at the time that wounds should be allowed to dry out and form scabs to promote healing, wounds heal faster if kept moist (Winter et al 1962). Since then, the use of "cheap" gauze dressings and bandages has given way to more technically advanced dressings, such as foams and hydrofibres, which interact with and manage the wound environment. The use of these now established dressings in combination with the "gold standard" high compression therapy is generally effective in the management of VLUs, although up to $20 \%$ of VLUs fail to heal despite being treated with this regime (White 2006).

The reasons for the lack of response to treatment of hardto-heal VLUs have not yet been fully elucidated, although scientific and clinical research indicates that, instead of progressing through the four distinct but overlapping phases of healing (hemostasis, inflammation, proliferation, and remodeling), these wounds become 'stuck' in a prolonged inflammatory phase (Timmons 2006).

Control of the processes within the phases of wound healing is complex and involves the interaction of several different cell types (eg, neutrophils, lymphocytes, macrophages, fibroblasts), regulatory mediators (eg, cytokines, growth factors), extracellular matrix (ECM) components (eg, fibronectin, fibrin, collagen, elastin, laminin, proteoglycans, glycosaminoglycans), proteases and their inhibitors (eg, matrix metalloproteinases [MMPs] and tissue inhibitors of metalloproteinases [TIMPs]) (Schultz et al 2005). Early in the wound healing process there is a requirement for the synthesis and deposition of ECM proteins such as fibrin and fibronectin (Hodde and Johnson 2007) and a sub-set of matrix proteins that are expressed transiently during the wound healing process, eg, galectins, osteopontin, SPARC, syndecans, tenascins, thrombospondins, and vitronectin (Agren and Werthen 2007). All of these matrix proteins form a provisional wound matrix which provides a scaffold that directs cells into the wound as well as stimulating them to proliferate, differentiate and synthesise new ECM to facilitate the proliferation and remodeling phases of the healing process (Schultz et al 2005).

In many chronic wounds, however, the normal healing process is disrupted by extensive tissue damage accompanied by biochemical and cellular imbalances, or by an underlying pathological state (eg, venous insufficiency) that can impair or even prevent healing. For example, in the case of hard-to-heal
VLUs, venous hypertension causes disturbed microcirculation and pathological changes to capillaries, which ultimately locks the condition in a self-amplifying cascade with persistent elevated levels of pro-inflammatory cytokines and proteases that appear to degrade the ECM components, growth factors and receptors that are essential for healing (Agren et al 2000; Schultz et al 2005) The ECM is a vital component of the healing process; intact or fragmented ECM molecules play a central role in modulating cells through transduction of a variety of signaling mechanisms (Agren et al 2000). Additionally, ECM plays a role in angiogenesis, recruitment of circulating progenitor cells, rapid scaffold degradation and constructive remodeling of damaged or missing tissues (Badylak 2002). In studies investigating the mechanisms that lead to venous leg ulceration, it has been demonstrated that intrinsic ECM degradation processes, prevalent in this disease state, are caused by the MMP family of enzymes (Herouy et al 2000; Bogaczewicz et al 2005). Specifically, it has been shown that fibronectin is absent in the base of nonhealing ulcers (Herrick et al 1992) although fibroblasts in such wounds synthesize fibronectin normally (Herrick et al 1996). It is hypothesized, therefore, that the reduced levels of fibronectin in nonhealing ulcers is due to excessive degradation of fibronectin by proteases in the ulcers, rather than decreased synthesis (Agren and Werthen 2007). On this basis, there is a clear need for new and advanced interventions that can interact with hard-to-heal VLUs that are locked in the inflammatory phase of the healing process and progress them to the subsequent proliferative stage of the process.

A specific focus of attention for the development of advanced wound therapies should be (and has been in recent years) the ECM, either by inducing its synthesis through growth factors, preventing its damage with sacrificial proteins or replacing it with autologous/homologous ECM proteins or their like. One mechanism by which this can be achieved is to provide hard-to-heal ulcers with surrogate ECMs which, although not homologous to the patient's own ECM, will provide transient structures that allow cellular adhesion and facilitate tissue regeneration by advancing the wounds to the proliferative phase of the healing process. Amelogenin has been identified as an ECM biocompatible protein that can be used as a surrogate scaffold protein. At physiological conditions, amelogenin self-assembles into globular aggregates up to micron-sizes. When applied to the wound bed it provides a temporary matrix for cell attachment and promotes wound healing. Amelogenin has been used successfully to treat patients with hard-to-heal VLUs and other chronic wound types. A summary of the experimental and clinical data relating to amelogenin is presented below. 


\section{In vitro studies}

Amelogenin has been used in periodontal applications for some time and there a number of experimental and clinical studies supporting its use in this application. Amelogenin has been used as a therapy for the regeneration of alveolar bone by inducing the formation of acellular extrinsic fiber cementum (Kim et al 2005). This protein has also been used to aid in soft tissue root coverage where it has been shown to enhance cellular activity during healing and improve periodontal regeneration (Giannobile and Somerman 2003; Young 2003). More recently, it has been shown that the combination of amelogenin and platelet-derived growth factor-BB (PDGF-BB) stimulated proliferation of primary human periodontal ligament fibroblasts and caused enhanced wound fill in an in vitro model (Chong et al 2006).

Experimental studies designed to elucidate the mechanism by which amelogenin works have shown that it interacts with a variety of cells and chemical messengers involved in the wound healing process. The studies indicate that amelogenin can reduce levels of pro-inflammatory cytokines (Myhre et al 2006).

One of the main cells involved in the healing process is the fibroblast. This cell synthesizes matrix components (eg, collagen) that are designed to fill the wound cavity and a variety of cell signals that modulate many different cellular responses such as angiogenesis and epithelialization. In experimental studies, amelogenin has been shown to:

- augment fibroblast-driven collagen matrix remodeling, increase dermal contraction and fibroblast proliferation (Grayson et al 2006)

- increase synthesis of growth factors, eg, transforming growth factor- $\beta 1$ (Grayson et al 2006) and vascular endothelial growth factor (Mirastschijski et al 2004)

- convert chronic fibroblasts into acute fibroblast phenotypes, and the restitution of proliferation and chemokine expression favoring an acute inflammatory response.

Amelogenin has also been shown to be effective in inducing an angiogenic response in a variety of in vitro models (Yuan et al 2003; Schlueter et al 2007; Ågren and Kleinman 2007). Angiogenesis, a key component of the healing process, is significantly reduced in the chronic wound (Drinkwater et al 2002; Ulrich et al 2005), thereby affecting the healing response.

\section{Clinical evaluations}

\section{Venous ulcers}

The fact that amelogenin can be used as a temporary ECM protein scaffold provides a sound basis for its use in the treatment of wounds, particularly chronic wounds that are deficient in normal ECM components. It was, therefore, proposed that amelogenin could be used to treat hard-to-heal VLUs. Initially, a preliminary clinical study was undertaken to ascertain the optimum number of applications of amelogenin (Xelma ${ }^{\circledR}$, Molnlycke Health Care, Gothenburg, Sweden) that are required to be applied to a VLU to produce a healing response. The study compared once weekly applications of amelogenin in conjunction with high compression therapy to the hard-to-heal VLUs (duration $>6$ months) of patients over 3, 6 and 12 week periods (Romanelli et al 2006) The wounds were assessed every second week up to week 8 , at final visit (week 12), and at follow-up visit (12 weeks after the final visit). Ulcers were traced and reduction in size was determined by using digital planimetry. The results showed that patients who received the 12-weeks treatment had a larger ulcer reduction $(-72 \%)$ compared with patients who received the 3 or 6 -weeks (-22 and $-48 \%$ respectively) treatment with amelogenin (Figure 1 and Table 1).

The results of this preliminary study showed that hard-toheal VLUs elicited a positive healing response to a defined period (ie, 12 weeks) treatment with amelogenin. Using this information, a single-blinded, randomized, multi-centre study involving 117 patients was undertaken to assess the effect of amelogenin on hard-to-heal VLUs (Vowden et al 2006).

To satisfy the inclusion criteria for the study, patients had to have a VLU with a size between 5 and $25 \mathrm{~cm}^{2}$ and duration of at least six months that had been treated with controlled compression therapy for at least one month prior to enrolment. Patients were randomised to receive either amelogenin $\left(X_{e}\right)^{\circledR}{ }^{\circledR}$, Molnlycke Health Care, Gothenburg, Sweden) plus high compression $(n=62)$ or a control treatment consisting of a placebo gel (the amelogenin carrier vehicle) plus high compression $(n=61)$. The amelogenin was applied weekly under secondary dressings for up to a maximum of 12 weeks. Although no statistical differences could be identified between the groups as a whole, the percentage wound size reduction was greater in the group treated with amelogenin. A more detailed sub-group analysis was undertaken on patients with ulcers of greater than $10 \mathrm{~cm}^{2}$ at baseline and for ulcers of duration greater than 6 months. The results of this analysis demonstrated that there was a difference in percentage wound reduction in the group treated with amelogenin (33.8\% vs $25.6 \%$, respectively). It was highlighted that this difference was greatest for the group of patients $(\mathrm{n}=61)$ with the larger ulcers $\left(>10 \mathrm{~cm}^{2}\right)$ (amelogenin 25\% vs control 7.9\%) (Figure 2). 


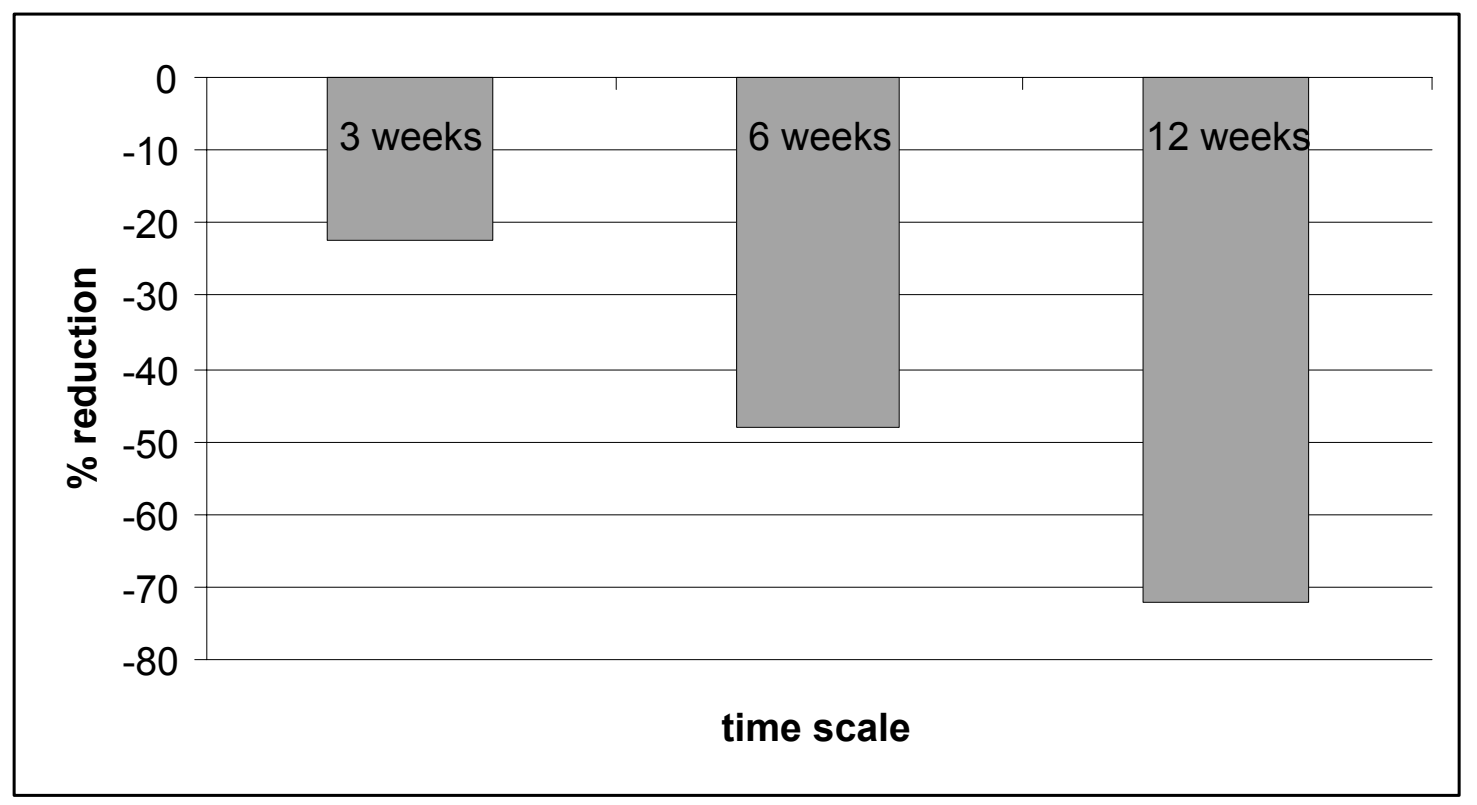

Figure I Percentage change in VLU size following 3,6 and I2 weeks of treatment with amelogenin. Copyright (C) 2006. Reproduced with permission from Romanelli M, Ellervee $\mathrm{T}$, Jarve $\mathrm{H}$, et al 2006. Amelogenins $\left(\mathrm{Xelma}^{\circledR}\right)$ in hard-to-heal venous leg ulcers, an open regime investigation [poster]. European Wound Management Association Conference, Prague, Czech Republic.

Overall the results of this study showed that amelogenin was well tolerated by the patients, with a trend towards less pain and a reduction in the volume of exudate produced by the ulcers in favor of the group treated with amelogenin. This study highlighted the problems associated with undertaking clinical trials involving VLUs, the main ones being the wide variations in wound demographics (eg, sizes and ages) and their response to treatments. In future clinical studies, this might be overcome by stratifying the wounds accordingly. In addition, there was a significant amount of variation between the pre-study treatment regimens employed at different centers, a phenomenon which if not accounted for by allowing a run-in period, may alter the

Table I Percentage change inVLU size following 3,6 and I 2 weeks of treatment with amelogenin. Copyright (C) 2006. Reproduced with permission from Romanelli M, Ellervee T, Jarve H, et al 2006.Amelogenins $\left(\mathrm{Xelma}^{\circledR}\right)$ in hard-to-heal venous leg ulcers, an open regime investigation [poster]. European Wound Management Association Conference, Prague, Czech Republic

\begin{tabular}{lll}
\hline Regimen & \multicolumn{2}{l}{ \% Change in ulcer size } \\
\cline { 2 - 3 } & Median & [Q। Q3] \\
\hline 3 weeks, $\mathrm{n}=10$ & -22.4 & {$[-72.5-15.3]$} \\
6 weeks, $\mathrm{n}=7$ & -48 & {$[-82.1-31.3]$} \\
12 weeks, $\mathrm{n}=12$ & -72 & {$[-83.90 .3]$} \\
\hline
\end{tabular}

outcome of a clinical trial. As such, the authors concluded that amelogenin could be clinically useful in the treatment of patients with ulcers that could be classified as hard-toheal (eg, of duration longer than 6 months and a size greater than $10 \mathrm{~cm}^{2}$ ).

Another clinical trial was undertaken using the lessons learnt from the previous study: this was an open, randomized, comparative, parallel group, multi-centre study involving patients with hard-to-heal VLUs (Vowden et al 2007). The inclusion criteria incorporated ulcer size of greater than $10 \mathrm{~cm}^{2}$ and duration of longer than 6 months, both of which have previously been reported in the literature as prognostic indicators of venous ulcers that are unlikely to respond to treatment and may be classed as hard-to-heal (European Wound Management Association 2002). The primary objective of this study was to compare the results of 12 consecutive weeks of treatment with amelogenin (Xelma ${ }^{\circledR}$, Molnlycke Health Care, Gothenburg, Sweden) plus high compression versus compression therapy alone in hard-to-heal VLUs. Percentage reduction of wound size from baseline, the percentage of improved ulcers, and parameters such as pain related to the disease, pain at dressing changes and the amount and nature of exudate were evaluated. The safety and tolerability of amelogenin were determined in relation to recorded adverse events. Eligibility for inclusion included adult, mobile patients with 'hard-to-heal' VLUs 


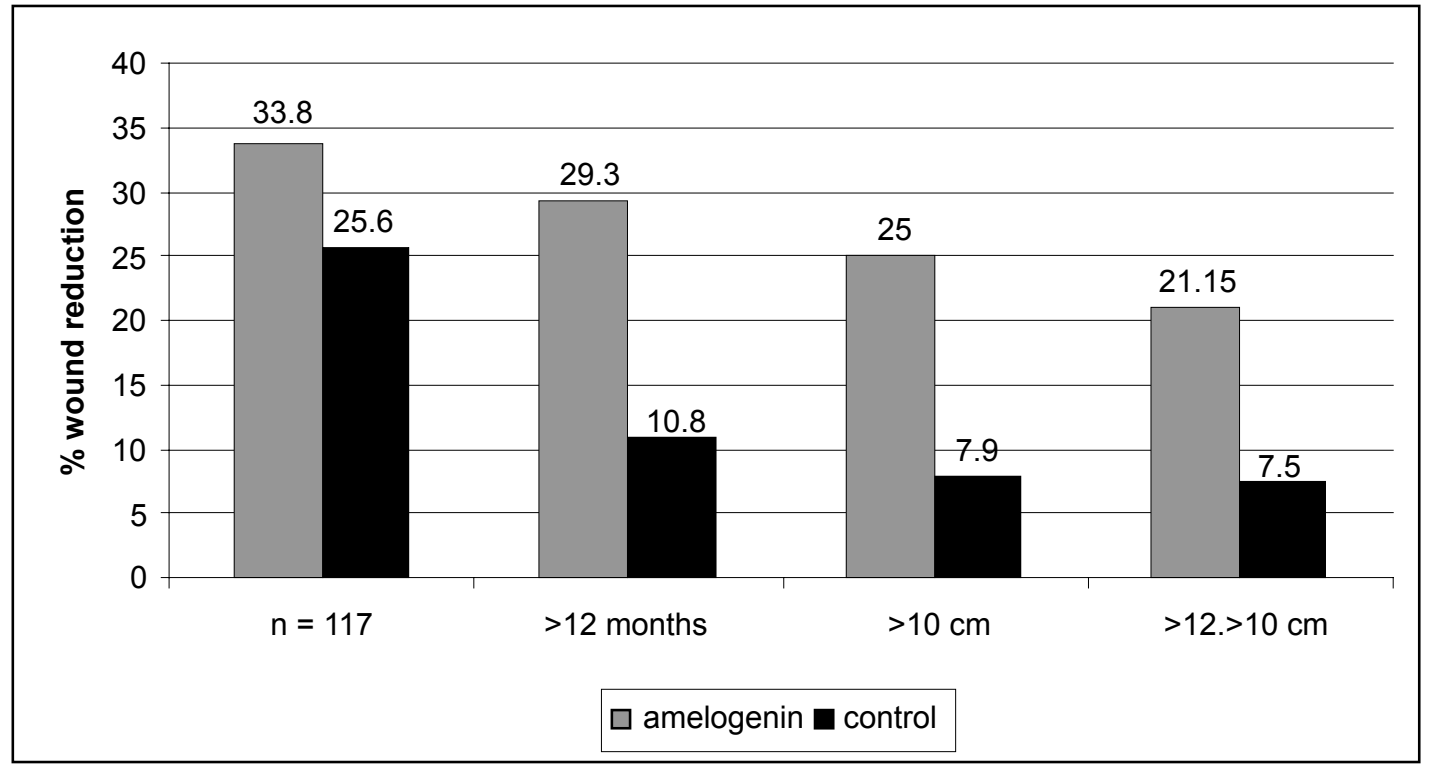

Figure 2 Median wound size reduction in the ITT population and sub-group ITTs following treatment with amelogenin and control. Copyright (C) 2006. Reproduced with permission from Vowden P, Romanelli M, Peter R, et al 2006. The effect of amelogenins (Xelma ${ }^{\circledR}$ ) on hard-to-heal venous leg ulcers. Wound Rep Reg, I4:243-6.

that had been treated with compression therapy for at least 1 month prior to screening. The ulcers had to be at least 6 months old, with a surface area at inclusion of at least $10 \mathrm{~cm}^{2}$, but not exceeding $30 \mathrm{~cm}^{2}$, and not demonstrating excessive exudate or signs of infection. At the end of the run in period additional criteria for eligibility, eg, change in wound area (increase/decrease) of greater than or equal to $50 \%$ and a wound area between 8 and $36 \mathrm{~cm}^{2}$ were applied. In total, 83 patients were randomized to receive either amelogenin plus high compression bandaging $(\mathrm{n}=42)$ or high compression bandaging (control) alone $(\mathrm{n}=41)$. All participants received high compression bandaging therapy one month prior to, during the investigational period of 3-weeks run-in and throughout the 12 weeks of active treatment

The results of this study demonstrated that the amelogenin group showed a greater percentage reduction in ulcer size (mean $-33.11 \%$ ) compared to the control group (mean-11.07\%) from baseline to the last visit $(\mathrm{p}=0.03)$. The number of ulcers showing an improvement was significantly greater $(p=0.01)$ in the amelogenin treated group than the control group. Statistically significant differences in favor of the amelogenin group were also found the proportion of patients with none or low levels of exudate $(p=0.01)$.

Pain was evaluated in this study as related to the disease or to the ulcer. It was evaluated as part of the protocol by interviewing the patients who were asked to rate their pain on a scale with 11 steps; from $0=$ no pain to $10=$ unbearable pain
(Hartrick et al 2003). The results showed that a reduction in pain related to the disease and a reduction in pain at dressing change were more apparent in the amelogenin treated group. Statistical analysis showed that the amelogenin group had significantly $(\mathrm{p}=0.01)$ greater ulcer pain reduction. The mean and $95 \% \mathrm{CI}$ for the difference between the amelogenin and the control group at final visit was $-1.59(-2.84-0.34)$. Pain is considered to be a significant problem in relation to patients with VLUs, and has been highlighted as cause for major concern when treating patients (Price et al 2007). The European Wound Management Association (2002) has developed a position document that provides clinical recommendations to assess and manage wound pain, especially at dressing change. Pain management has therefore become a major part of wound care with many organizations and care providers incorporating pain management into standards, guidelines and clinical practice (Price et al 1997; Young 2007). Thus the fact that amelogenin therapy, in a way as yet undetermined, was shown to significantly reduce the pain that patients with hard-to-heal VLUs experienced is noteworthy and perhaps requires further investigation.

As well as the study participants having hard-to-heal wounds, the population evaluated represents possibly the "worst case scenario" patients, eg, some patients within the amelogenin group had very old ulcers (10 years or more). This highlights the potential advantages of using advanced therapies for the treatment of ulcers within this patient 
population. The safety of amelogenin has been established by completing the extensive testing that is required for medical devices. The safety profile of amelogenin is underlined by the results of this study with few adverse events reported, and no significant difference between the treatment and control groups.

A series of case studies has been presented in which a number of patients with hard-to-heal VLUs were treated with amelogenin $\left(X_{\text {elma }}{ }^{\circledR}\right.$, Molnlycke Health Care, Gothenburg, Sweden) therapy (Huldt-Nystrom et al 2007). The healing of these wounds was evaluated by comparing the wound area at baseline (before treatment) and upon completion of treatment. In this study, the amelogenin was applied to a clean wound bed, on a weekly basis, up to a maximum of 12 weeks in conjunction with standard compression therapy. Some of these patients were treated with compression for four weeks prior to being included in the evaluations, in order to ensure that ulcers were truly nonhealing. The results showed that the success rate with amelogenin therapy was high, with approximately $80 \%$ of the ulcers either healing $(60 \%)$ or showing a reduction in ulcer size $(20 \%)$ after treatment. Only two ulcers (10\%) remained unchanged and two ulcers deteriorated $(10 \%)$ during the treatment period. Pseudomonas colonization/infection was present in some wounds after treatment had been started, but this was treated with appropriate antibiotic therapy, and did not affect the successful healing outcome of the wounds.

A small study in the United Kingdom (Acton 2007) has demonstrated similar results in that amelogenin $\left(\mathrm{Xelma}^{\circledR}\right.$, Molnlycke Health Care, Gothenburg, Sweden) stimulated a healing response. Even if the wounds did not progress to complete healing, they were significantly healthier than before the amelogenin treatment had started and were able to go on to be grafted. In addition, an improvement in quality of life was observed in the patients who received amelogenin therapy.

Another study undertaken in the United Kingdom evaluated amelogenin for the treatment of patients $(n=17)$ with a variety of complex hard-to-heal ulcers, including rheumatoid ulcers $(\mathrm{n}=2)$, wounds complicated by rheumatoid arthritis $(n=3)$, neuropathic foot ulcers $(n=4)$, venous ulcers $(n=4)$, and a single ulcer of mixed etiology. The results from this study followed a similar trend to that seen in the treatment of other hard-to-heal ulcers, in that an early reduction in wound pain and exudate were apparent. Overall, six wounds healed after a mean of 8 applications of (Xelma ${ }^{\circledR}$, Molnlycke Health Care, Gothenburg, Sweden) (range 3-16 applications), six patients showed an improvement of a greater than
$50 \%$ reduction in ulcer size, and were continuing to receive amelogenin therapy. Only two patients were discontinued due to infection and wound deterioration (Vowden et al 2007a).

In a case study series involving eight patients with a total of 10 VLUs (mean duration of 9.3 years), amelogenin (Xelma ${ }^{\circledR}$, Molnlycke Health Care, Gothenburg, Sweden) was applied weekly and covered with a secondary dressing. Treatment duration with amelogenin was 12 weeks and patients were followed up for a period of 24 weeks. An overall healing rate of $50 \%$ was reported, including one patient with a wound duration of 65 years. The other ulcers were in a healing state at 24 weeks, with a mean reduction in size of $60 \%$ compared with baseline values. Exudate levels and wound odor also reduced over the treatment period (Hampton et al 2007).

\section{Diabetic ulcers}

Amelogenin has recently been evaluated in a study consisting of a mixed population of patients with both diabetic foot ulcers $(n=5)$ and VLUs $(n=5)$. Both sets of patients demonstrated initiation of healing with a high percentage (80\%) of ulcers healing completely (Meuleneire 2007). Diabetic foot ulcers have a different aetiology to that of VLUs, they are a significant complication of diabetes mellitus and often precede lower-extremity amputation. The most frequent underlying aetiologies are neuropathy, trauma, deformity, high plantar pressures, and peripheral arterial disease. The primary treatment is usually offloading to reduce pressure to the sensitive areas and then management of the wound with appropriate dressings. Infection and high levels of wound exudate may be complicating factors in the treatment of these wounds, but the early indications are that amelogenin therapy, in conjunction with appropriate wound management techniques, can be beneficial.

\section{Pyoderma gangrenosum}

Pyoderma gangrenosum (PG) is an inflammatory ulcerative condition of unknown etiology, although an autoimmune mechanism including immune complex-mediated neutrophilic vascular reactions has been suggested. PG is frequently associated with various diseases, but up to $50 \%$ of cases are idiopathic. It is a disease that causes tissue to become necrotic, causing deep ulcers that usually occur on the legs. When they occur, they can lead to chronic wounds. There are two main types of ulcers that occur: the normal ulcerative form which occurs on the legs, and an 'atypical' form that is more superficial and occurs on the hands and other parts of the body. Though the etiology is not well understood, the disease is thought to be due to immune system dysfunction 
and, in particular, improper functioning of neutrophils. At least half of all PG patients also suffer from illnesses that affect their systemic function. Classical ulcerative PG is characterized by the appearance of nodules with pustules that enlarge and lead to chronic ulcers violaceous and undermined borders (Dini et al 2007) Amelogenin therapy has been used to treat two female patients with recalcitrant PG of the lower leg lasting an average of 11 months (Dini et al 2007a). The treatment was applied weekly under an occlusive dressing for a maximum of 8 weeks in conjunction with systemic immunosuppressive therapy before and during the topical treatment. The lesions improved, showing advances in granulation tissue formation and wound size reduction (Figures 3a-c). Additionally, pain control was reported and the therapy was well-tolerated with no adverse effects.

The clinical evidence suggests that in order for advanced therapies like amelogenin to be cost effective in for example the treatment of VLUs, then suitable patients must be carefully identified and according to prognostic indicators of delayed healing such as wound size (larger than $10 \mathrm{~cm}^{2}$ ) and wound age (duration of greater than 6 months).
Additionally, if wounds do not show a decrease in size of $40 \%$ or more during 4 weeks of compression therapy, then these wounds can be identified as hard-to-heal (Phillips et al 2000) and consideration should be given to their treatment with advanced therapies such as amelogenin. Compression therapy in conjunction with amelogenin is thought to be an essential adjunctive treatment. This is because it treats the underlying cause of venous hypertension and enables blood to be returned from the lower limbs, thereby reducing oedema and the consequences of stasis.

Infection in the wound must be treated prior to application of amelogenin, although it has been seen that concurrent treatment of infection with antibacterial therapy is not detrimental to the amelogenin protein as a therapeutic agent. Additionally, wounds that exudate highly do not appear to benefit as well from amelogenin therapy as wounds that have low to moderate levels of exudate, implicit in this therefore is the requirement that exudate should be managed with compression and appropriate dressings that can absorb the fluid. Wound bed preparation is of vital importance. Dead, sloughy and necrotic tissue is prevalent in chronic venous

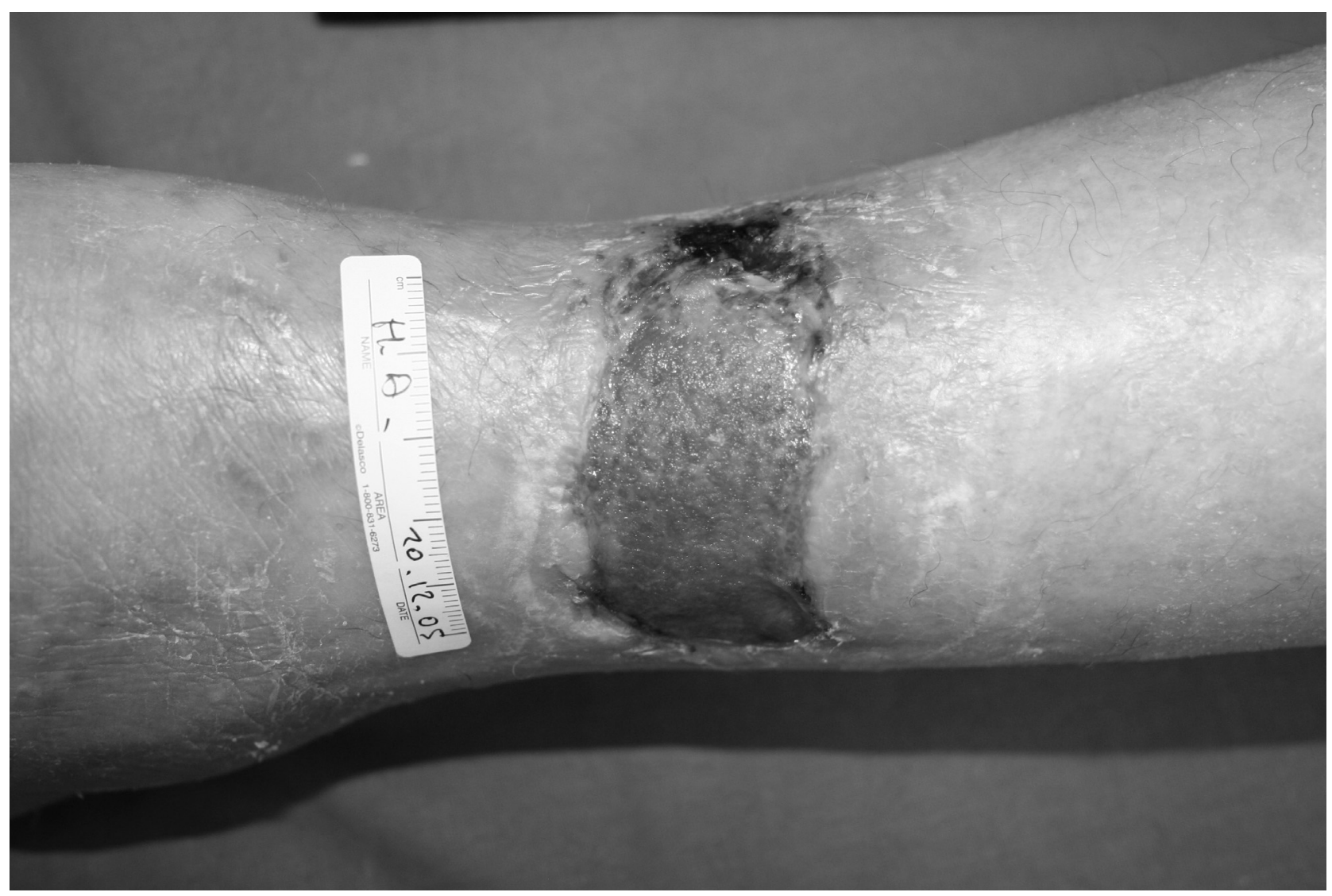

Figure 3a Pyoderma gangrenosum at baseline before amelogenin application. 


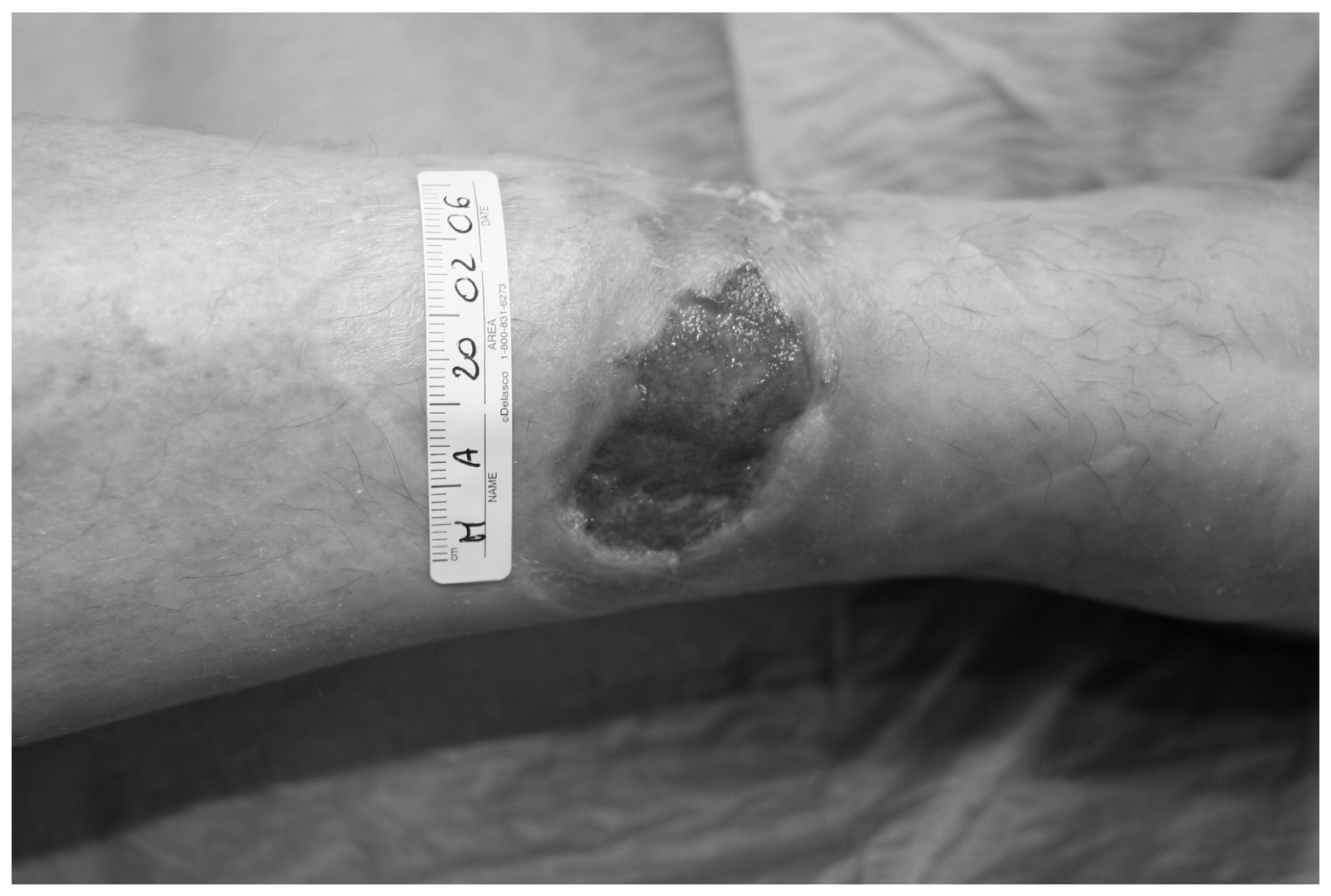

Figure 3b Same lesion after eight weeks of amelogenin treatment.

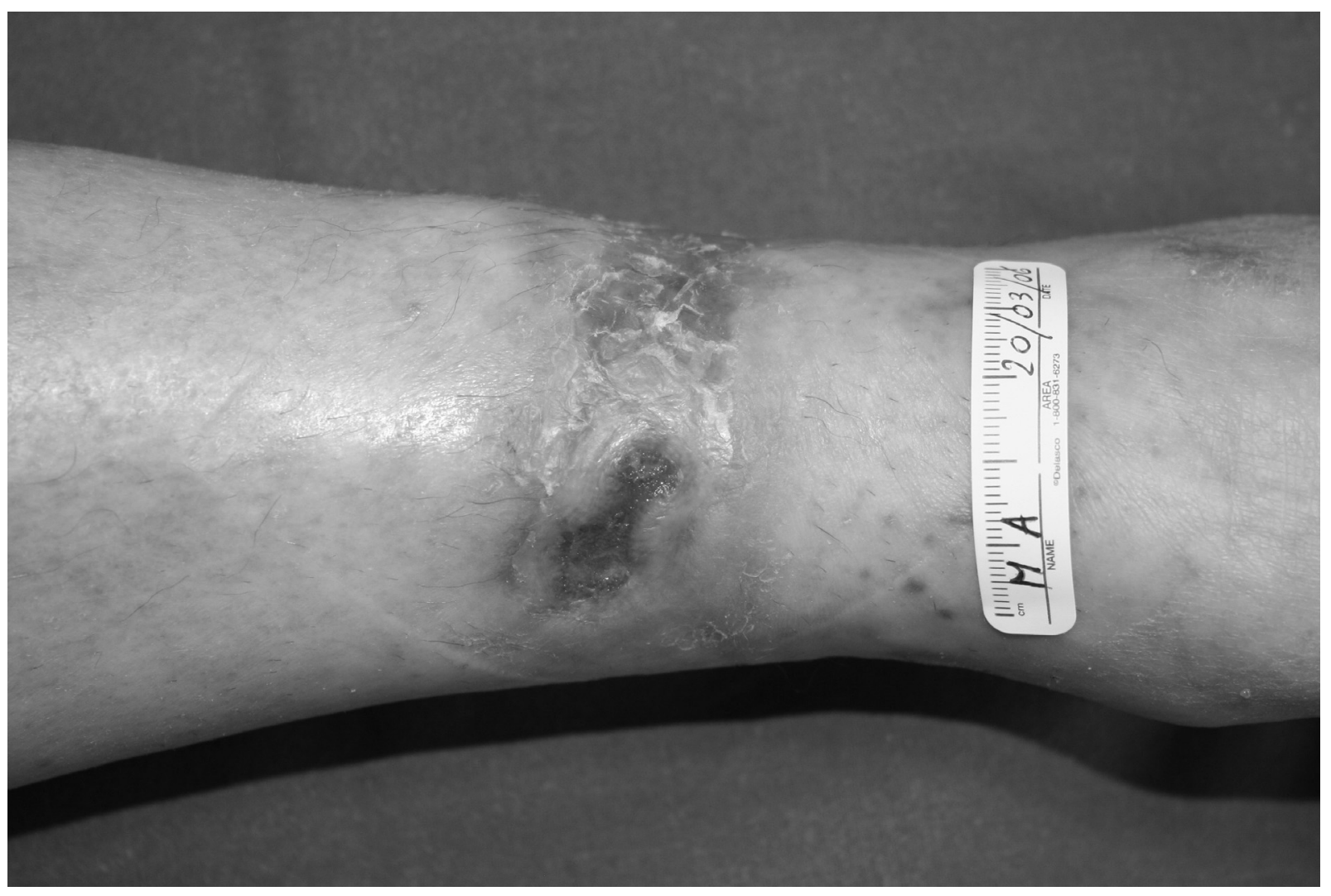

Figure 3c Same lesion at 12 weeks follow up. 
ulcers. This tissue impedes the growth of healthy granulation tissue and re-epithelisation and therefore must be removed. This is standard practice in the treatment of such wounds. It is also thought that the presence of such dead tissue may prevent the amelogenin protein integrating into the fabric of the wound bed and thus providing the temporary scaffolding to which cells can attach and subsequently stimulate the healing process.

Qualitatively, VLUs treated with amelogenin appear to heal much better than with high compression alone, with no evidence of hypertrophic scarring or excessive wound contracture.

\section{Conclusions}

There is a raft of experimental evidence that supports a mechanism for amelogenin in facilitating the wound healing process. For example, it appears that amelogenin provides a temporary scaffold to allow cellular adherence of fibroblasts and epithelial cells. This in turn will stimulate various functions of cells with regards to the wound healing process, eg, migration (into the wound), proliferation (increasing the number of cells and filling the wound), and synthesis of mediators (growth factors and cytokines) that can regulate processes that are relevant to wound healing such as angiogenesis.

This review highlights that a number of well controlled trials have established that amelogenin treatment has a statistically beneficial effect in the clinical environment. It has been shown that amelogenin can significantly reduce ulcer size and also significantly increase the number of patients showing a greater than $50 \%$ reduction in wound size, compared with comparator controls. Pain is a problem in patients with VLUs, but it has been shown that amelogenin therapy can reduce the level of pain experienced by patients with hard-to-heal VLUs, compared with comparator controls. It is interesting to conjecture whether this is simply due to healing of the wound or whether amelogenin has a direct remedial effect upon pain, future studies may elucidate this mechanism.

\section{References}

Acton C. 2007. The evaluation of an advanced treatment (Xelma) on hardto-heal leg ulcers that have not responded to standard therapy [poster] European Wound Management Association Conference, Glasgow, United Kingdom.

Ågren MS, Eaglstein WH, Ferguson MW, et al. 2000. Causes and effects of the chronic inflammation in venous leg ulcers. Acta Derm Venereol Suppl (Stockh), 210:3-17.

Ågren MS, Kleinman HK. 2007. Effect of amelogenins on angiogenesis ex vivo. Wound Rep Reg, 14:A74.

Ågren MS, Werthén M. 2007. The extracellular matrix in wound healing: a closer look at therapeutics for chronic wounds. Int J Low Extrem Wounds, 6:82-97.
Anderson I. 2006. Aetiology, assessment and management of leg ulcers. Wound Essentials, 1:20-36.

Badylak SF. 2002. The extracellular matrix as a scaffold for tissue reconstruction. Semin Cell Dev Biol, 13:377-83.

Bogaczewicz J, Dudek W, Wronski J, et al. 2005. [The role of matrix metalloproteinases in venous leg ulcers development]. Pol Merkur Lekarski, 19(113):686-92.

Chong CH, Carnes DL, Moritz AJ, et al. 2006. Human periodontal fibroblast response to enamel matrix derivative, amelogenin, and platelet-derived growth factor-BB. J Periodontol, 77:1242-52.

Dini V, Romanelli M, Bertone MS, et al. 2007. Improvement of idiopathic pyoderma gangrenosum during treatment with anti-tumor necrosis factor alfa monoclonal antibody. Int J Low Extrem Wounds, 6:108-13.

Dini V, Bertone MS, Barbanera S, et al. 2007a. The use of amelogenin in pyoderma gangrenosum [poster]. European Wound Management Association Conference, Glasgow, United Kingdom.

Drinkwater SL, Burnand KG, Ding R, et al. 2003. Increased but ineffectual angiogenic drive in nonhealing venous leg ulcers. J Vasc Surg, 38:1106-12.

European Wound Management Association. 2002. Position Document: Pain at wound dressing changes. London: Medical Education Partnership.

Giannobile WV, Somerman MJ. 2003. Growth and amelogenin-like factors in periodontal wound healing. A systematic review. Ann Periodontol, 8:193-204.

Grayson RE, Yamakoshi Y, Wood EJ, et al. 2006. The effect of the amelogenin fraction of enamel matrix proteins on fibroblast-mediated collagen matrix reorganization. Biomaterials, 27:2926-33.

Hampton S, Kerr A, Bree-Aslan C. 2007. An evaluation of a matrix replacement treatment in intractable wounds. Poster presentation. European Wound Management Association Conference, Glasgow, United Kingdom.

Hartrick CT, Kovan JP, Shapiro S. 2003. The numeric rating scale for clinical pain measurement: a ratio measure. Pain Pract, 3:310-16.

Herouy Y, Trefzer D, Zimpfer U, et al. 2000. Matrix metalloproteinases and venous leg ulceration. Eur J Dermatol, 10:173-80.

Herrick SE, Sloan P, McGurk M, et al. 1992. Sequential changes in histologic pattern and extracellular matrix deposition during the healing or chronic venous ulcers. Am J Pathol, 141:1085-95.

Herrick SE, Ireland GW, Simon D, et al. 1996. Venous ulcer fibroblasts compared with normal fibroblasts show differences in collagen but not fibronectin production under both normal and hypoxic conditions. J Invest Dermatol, 106:187-93.

Hodde JP, Johnson CE. 2007. Extracellular matrix as a strategy for treating chronic wounds. Am J Clin Dermatol, 8:61-6.

Hofman D, Ryan TJ, Arnold F, et al. 1997. Pain in venous leg ulcers. $J$ Wound Care, 6:222-4.

Huldt-Nystrom T, Bonesronning JH, Dofour N, et al. 2007. Treatment of hard-to-heal chronic venous ulcerations with amelogenin. An update on the Norwegian experience [poster]. European Wound Management Association Conference, Glasgow, United Kingdom.

Kim NH, Tominaga K, Tanaka A. 2005. Analysis of eosinophilic round bodies formed after injection of enamel matrix derivative into the backs of rats. J Periodontol, 76:1934-41.

Mekkes JR, Loots MA, van der Wal AC, et al. 2003. Causes, investigation and treatment of leg ulceration. Br J Dermatol, 148:388-401.

Meuleneire F. 2007. A preliminary evaluation of an advanced therapy $\left(X \mathrm{Xlm}^{\circledR}\right)$ for the treatment of hard to heal wounds: venous leg ulcers and diabetic foot ulcers [poster]. European Wound Management Association Conference, Glasgow, United Kingdom.

Mirastschijski U, Konrad D, Lundberg E, et al. 2004. Effects of a topical enamel matrix derivative on skin wound healing. Wound Rep Reg, $12: 100-8$.

Myhre AE, Lyngstadaas SP, Dahle ML, et al. 2006. Anti-inflammatory properties of enamel matrix derivative in human blood. J Periodontal Res, 41:208-13. 
Phillips T, Machado F, Trout R, et al. 2000. Prognostic indicators in venous ulcers. J Am Acad Dermatol, 43:627-30.

Price P, Fogh K, Glynn C, et al. 2007. Managing painful chronic wounds: the Wound Pain Management Model. Int Wound J, 4(Suppl 1):4-15.

Romanelli M, Ellervee T, Jarve H, et al. 2006. Amelogenins (Xelma ${ }^{\circledR}$ ) in hard-to-heal venous leg ulcers, an open regime investigation [poster]. European Wound Management Association Conference, Prague, Czech Republic.

Ryan S, Eager C, Sibbald RG. 2003. Venous leg ulcer pain. Ostomy Wound Manage, 49(4 Suppl):16-23.

Schlueter SR, Carnes DL, Cochran DL. 2007. In vitro effects of enamel matrix derivative on microvascular cells. J Periodontol, 78:141-51.

Schultz GS, Ladwig G, Wysocki A. 2005. Extracellular matrix: review of its roles in acute and chronic wounds [online]. Accessed June 13, 2007. URL: http://www.worldwidewounds.com/2005/august/.

Soon K, Acton C. 2006. Pain-induced stress: a barrier to wound healing. Wounds UK, 2:92-101.

Timmons J. 2006. Skin function and wound healing physiology. Wound Essentials, 1:8-17.

Ulrich D, Lichtenegger F, Unglaub F, et al. 2005. Effect of chronic wound exudates and MMP-2/-9 inhibitor on angiogenesis in vitro. Plast Reconstr Surg, 116:539-45.

Vowden P, Romanelli M, Peter R, et al. 2006. The effect of amelogenins $\left(\mathrm{Xelma}^{\circledR}\right)$ on hard-to-heal venous leg ulcers. Wound Rep Reg, 14:243-6.
Vowden P, Romanelli M, Price P. 2007. Effect of amelogenin extracellular matrix protein and compression on hard-to-heal venous leg ulcers. $J$ Wound Care, 16:189-95.

Vowden K, Mcgowan J, Pilcher, M, et al. 2007a. Experience with the use of an amelogenin-based extracellular matrix substitute in the management of a variety of complex hard-to-heal chronic wounds [poster]. European Wound Management Association Conference, Glasgow, United Kingdom.

White R. 2006. Delayed wound healing: who, what, when and why? Nursing Standard, October 25(Suppl):47-54.

Winter GD. 1962. Formation of the scab and the rate of epithelialisation of superficial wounds in the skin of the young domestic pig. Nature, 193:293.

Young GR. 2003. Soft-tissue root coverage. Clinical rationale and case report. Dent Today, 22:134-9.

Young T. 2007. Assessment of wound pain: overview and a new initiative. Br J Nurs, 16:456-61.

Yuan K, Chen CL, Lin MT. 2003. Enamel matrix derivative exhibits angiogenic effect in vitro and in a murine model. J Clin Periodontol, 30:732-8. 